Quellen und Forschungen aus italienischen Archiven und Bibliotheken 



\section{Quellen und}

Forschungen aus

italienischen Archiven

und Bibliotheken

Herausgegeben vom

Deutschen Historischen Institut in Rom

$2019 \cdot$ Band 99 
Deutsches Historisches Institut in Rom

Via Aurelia Antica 391

00165 Roma

Italien

http://www.dhi-roma.it

ISSN 0079-9068

e-ISSN 1865-8865

Bibliografische Information der Deutschen Nationalbibliothek

Die Deutsche Nationalbibliothek verzeichnet diese Publikation in der Deutschen Nationalbibliografie; detaillierte bibliografische Daten sind im Internet über http://dnb.dnb.de abrufbar.

(c) 2020 Walter de Gruyter GmbH, Berlin/Boston Satz: Dörlemann Satz GmbH \& Co. KG, Lemförde Druck und Bindung: CPI Books GmbH, Leck @ Gedruckt auf säurefreiem Papier Printed in Germany

www.degruyter.com 


\section{Inhaltsverzeichnis}

Martin Baumeister

Jahresbericht des DHI Rom 2018 — IX

\section{Themenschwerpunkt}

Sprach- und ethnische Konflikte in Klöstern nördlich und südlich der Alpen zum Ausgang des Mittelalters und in der frühen Neuzeit. Drei Fallbeispiele

Andreas Rehberg/Gabriela Signori

Sprach- und ethnische Konflikte in Klöstern nördlich und südlich der Alpen zum Ausgang des Mittelalters und in der frühen Neuzeit. Eine Einführung - 3

Maximilian Alexander Trofaier

Konstruierte Fremdheit. Die Beziehungen der irischen Mönche des Schottenklosters in Wien zu ihrem Umfeld - 18

Petr Hlaváček

Ein Sprachenkonflikt unter den Franziskanern? Das Observantenvikariat „Bohemia“ vor 1500 und seine „nationalen“ Streitigkeiten — 27

Andreas Rehberg

Subiaco e Farfa contesi. L'espulsione dei monaci „tedeschi“ da due monasteri nei dintorni di Roma dopo il $1500-50$

\section{Artikel}

Wolf Zöller

Saeculum obscurum - der epigraphische Befund (ca. 890-1000) - 79

Paolo Tomei

Una nuova categoria documentaria nella Toscana marchionale: la donazione in forma di mandato. Cultura grafica e strutture politiche in una società di corte -115

\section{Stefania Zucchini}

Studenti, copisti, cuochi, panettieri. I tedeschi nel collegio studentesco della Domus Sapientiae di Perugia (sec. XV) -150 
Florian Runschke

Das Generalkommissariat in Italien von 1624 bis 1632 . Auftrag, Arbeit und Akzeptanz der ersten beiden Amtsinhaber — 201

Margherita Palumbo

L'Accademie de Filles de qualité di Luise von Hohenzollern-Hechingen - 243

Marie Bossaert

Italiani o Ottomani? La seconda generazione di immigrati italiani a Istanbul (Ottocento - primo Novecento) -262

Kordula Wolf

Orientalismo meridionale, patriottismo e musulmani nell'Italia medievale. Riflessioni sulla visione storica di Michele Amari e sulla sua influenza storiografica - 285

Francesco Zavatti

Appealing Locally for Transnational Humanitarian Aid. Italian Bishops and the Great Irish Famine - 313

Christiane Baumann

„La perduta gente“. Richard Voß’ (1850-1918) naturalistische Italienbilder und der Einfluss des Historikers und Essayisten Ferdinand Gregorovius - 340

Massimiliano Valente

Benedetto XV e l'Ufficio provvisorio per informazioni sui prigionieri di guerra (1914-1918) - 367

Matteo Stefanori

Le strade che portano a Roma. Ebrei stranieri nella capitale, 1933-1945.

Primi risultati di una ricerca in corso -387

Stefano Twardzik

Il memoriale di Aldo Moro. La ricerca di una ratio nella sequenza dei manoscritti in fotocopia rinvenuti nel $1990-428$

\section{Forum}

Jörg Hörnschemeyer „Möchten Sie das Programm wirklich löschen?“ Warum sich die Geisteswissenschaften mit der Nachhaltigkeit von Forschungssoftware auseinandersetzen sollten -491 


\section{Forschungsberichte}

\section{Riccarda Suitner}

A proposito di tre recenti studi su Riforma in Italia e minoranze religiose nella prima età moderna $\longrightarrow 505$

\section{Tagungen des Instituts}

Nastasia Sophie Tietze

Rethinking the Soundscape. Musical Events and the Soundscape of Italian Cities, XVI-XIX Century - 515

Bianca Gaudenzi

Mediterranean Empires of the Interwar Period. Biopolitics, Chronopolitics, Geopolitics - 520

Markéta Štědronská

„Man müßte nach Rom gehen“. Bernd Alois Zimmermann und Italien - 526

Circolo Medievistico Romano $2018-529$

\section{Nachrufe}

Arnold Esch

Rudolf Schieffer. 1947-2018 - 535

Arnold Esch

Brigide Schwarz. 1940-2019 537

\section{Rezensionen}

Leitrezension

Ronnie Po-chia Hsia

Understanding Global Catholicism in the Early Modern Era.

Christian Windler's important Study on Catholic Mission in Persia -541 
VIII

Inhaltsverzeichnis

Allgemein -544

Mittelalter -574

Frühe Neuzeit -664

19. -20. Jahrhundert -684

Verzeichnis der Rezensent/-innen - $\mathbf{7 2 0}$

Register der in den Rezensionen genannten Autor/-innen - 721 